FINANÇAS 


\title{
FXVOL: INDICADOR ANTECEDENTE DA TAXA DE CÂMBIO
}

\author{
FXVOL: ANTECEDENT INDICATOR OF THE EXCHANGE RATE
}

Vinicius Mothé Maia

Pontifícia Universidade Católica do Rio de Janeiro

André Luís Leite

Pontifícia Universidade Católica do Rio de Janeiro

Antonio Carlos Figueiredo

Pontifícia Universidade Católica do Rio de Janeiro
Marcelo Cabús Klötzle

Pontifícia Universidade Católica do Rio de Janeiro

Data de submissão: I 5 nov. 20 |5. Data de aprovação:

I0 mai. 2016. Sistema de avaliação: Double blind review. Universidade FUMEC / FACE. Prof. Dr. Henrique Cordeiro Martins. Prof. Dr. Cid Gonçalves Filho. Prof. Dr. Luiz Claudio Vieira de Oliveira

\section{RESUMO}

A forte correlação negativa entre a Taxa de Câmbio Brasil-EUA (Ptax) e o índice do mercado de ações (lbovespa) tem sido amplamente documentada na literatura acadêmica. $O$ índice de volatilidade cambial ( $F X v o l)$ representa a incerteza futura dos investidores em relação a taxa de câmbio um mês a frente. Essas duas evidências nos motivam a questionar qual o relacionamento entre $o$ FXvol e os retornos futuros da taxa cambial e do índice de mercado de ações, dado que o índice de volatilidade é visto como um termômetro da incerteza do investidor um período a frente. Investiga-se então a relação contemporânea entre o FXvol, a Ptax e o lbovespa, bem como a capacidade do FXvol de captar a possível relação entre o nível de incerteza presente no mercado e as variações relativas futuras da taxa de câmbio e do índice de ações. Os resultados encontrados apresentam evidências que o FXvol observado hoje descreve o comportamento da Ptax e do lbovespa para prazos futuros, atuando dessa forma como indicador antecedente dessas variáveis.

\section{PALAVRAS-CHAVE}

Índice de Volatilidade. FXvol. Taxa de Câmbio. Ptax e Ibovespa. 


\section{ABSTRACT}

The strong negative correlation between the Brazil-US Exchange Rate (Ptax) and the index of the stock market (Ibovespa) has been widely documented in the academic literature. The exchange rate volatility index ( $F X v o l)$ represents the future exchange rate uncertainty of investors regarding one month ahead. These two pieces of evidence motivate us to ask what the relationship between FXvol and future returns of the exchange rate and the stock market index, as the volatility index is seen as an investor uncertainty thermometer one period ahead. Investigating then the contemporary relationship between FXvol, the Ptax and the Ibovespa, and the FXvol's ability to capture the possible relationship between the present level of uncertainty in the market and future relative changes in the exchange rate and stock index. The findings provide evidence that the FXvol observed today describe the behavior of the Ptax and Ibovespa for future periods, thus acting as a leading indicator of these variables.

\section{KEYWORDS}

Volatility Index. FXvol. Exchange Rate. Ptax and Ibovespa.

\section{INTRODUÇÃO}

A volatilidade tem recebido especial atenção, com a consolidação da utilização dos derivativos financeiros, por parte dos profissionais que atuam nesse mercado e dos acadêmicos que o estudam. Esse interesse advém de a volatilidade ser uma variável, nos modelos de apreçamento de derivativos, que não se encontra disponível no mercado, razão pela qual se impõe a necessidade de mensurá-la. A busca por uma medida capaz de exprimir as incertezas do mercado, uma métrica segura e confiável, levou à criação dos Índices de Volatilidade por parte das bolsas de valores. A precursora do movimento de implantação desse tipo de índice no mercado financeiro mundial foi a Chicago Board Options Exchange (CBOE), com a criação do VIX, em 1993.

Em virtude de o VIX medir, segundo a segundo, a volatilidade implícita do mercado, Whaley (2000) aponta sua ampla utilização como medidor do medo dos investidores (investor fear gauge). $\mathrm{O}$ autor embasa sua afirmação ao notar que, em situações de estresse, as incertezas tendem a aumentar, levando os investidores a aumentar a taxa de desconto nas projeções do valor das ações. Consequentemente, ocorre uma queda nos preços do mercado acionário e um aumento da volatilidade captada pelo VIX.

Inspirada pela boa aceitação do VIX e sua ampla utilização, a BM\&FBovespa criou, em 2007, o Índice de Volatilidade Cambial do Dólar, FXvol. Sua elaboração foi pautada nos moldes do VIX, seguindo o trabalho de Demeterfi, Derman, Kamal et al. (1999). Objetivou-se, com sua concepção, criar um índice confiável e capaz de mensurar as incertezas em relação ao câmbio real/dólar, através das opções da moeda americana.

Sendo o FXvol, o medidor das incertezas futuras em relação a Ptax e, dada a 
correlação negativa significativa, registrada entre esta e o lbovespa, investiga-se, neste artigo, a relação contemporânea entre o FXvol, a Ptax e o lbovespa, bem como a capacidade do FXvol, de captar a possível relação entre o nível de incerteza presente no mercado e as variações relativas futuras da taxa de câmbio e do índice de ações.

A relevância da pesquisa se dá, em primeiro lugar, pela possibilidade de encontrar uma nova fonte de informação para pesquisadores e agentes de mercado. Segundo Xin (20I0), cada modelo de cálculo da volatilidade retém informações diferentes dos demais, podendo o FXvol agregar, assim, uma nova perspectiva da dinâmica de mercado ainda não percebida. Há uma escassez de trabalhos sobre o índice de volatilidade cambial brasileiro, e acreditase poder colaborar para gerar, sobre esse indicador, novas perspectivas que venham a ajudar na consolidação do mesmo, como uma referência de mercado.

A consolidação $F X$ vol se torna importante, pois Woo, Vicente e Barbedo (2009) apontam a falta de robustez em replicar a volatilidade da taxa de câmbio, utilizandose de instrumentos financeiros presentes atualmente no mercado. Uma consequência direta da ineficiência em se replicar essa volatilidade é a perda de um mecanismo relevante para se realizar, por exemplo, estratégias de hedge com $\circ$ intuito de se proteger das variações cambiais. Evidências atuais apontam para uma maior preocupação das organizações em se defender dessas variações, do que com fins especulativos, após a crise financeira de 2008 (COUTINHO; SHENG; LORA, 2012; LOPES; SCHIOZER; SHENG, 20I3).

Os resultados encontrados descrevem estatisticamente $\circ \mathrm{FXvol}$, analisam ques- tões de assimetria da variação relativa do índice em relação a retornos positivos e negativos da Ptax e do lbovespa e apresentam evidências de que o $\mathrm{FXvol}$ observado hoje, em média, descreve o comportamento da Ptax e do lbovespa para prazos futuros, atuando dessa forma como indicador antecedente dessas variáveis.

\section{REFERENCIALTEÓRICO}

A mensuração e previsão da volatilidade sempre foi uma preocupação de praticantes e acadêmicos, uma vez que estas não se encontram disponíveis no mercado para utilização nos modelos de apreçamento de ativos e derivativos. No que se refere ao mercado brasileiro, Gabe e Portugal (2004) destacam a superioridade dos modelos históricos sobre o modelo implícito. Entretanto, sugerem que o maior poder de previsão poderia se dar não devido à ineficiência dos métodos implícitos em predizer a volatilidade futura, mas a uma possível ineficiência do mercado.

Ainda a respeito da dicotomia tratada, Corrado e Miller Jr. (2006) procuraram avaliar qual das duas volatilidades explicaria melhor os retornos esperados. A pesquisa possibilitou concluir que a volatilidade implícita e os retornos esperados detinham uma correlação positiva e relevante, enquanto que a volatilidade histórica não alcançou resultados estatisticamente relevantes. A partir da relação entre a volatilidade implícita e retorno, as pesquisam avançaram sobre o comportamento dos índices de volatilidade, tendo como foco central dos estudos o VIX, precursor desses índices.

Whaley (2000) estudou a relação entre a queda no índice da bolsa de valores e o aumento do VIX. $\mathrm{O}$ autor indica que, em 
momento de crise, as taxas de desconto são revisadas para cima, acarretando uma redução do valor presente dos fluxos futuros aos acionistas e, por consequência, uma queda acentuada no valor dos ativos. Chen, $\mathrm{Da}$ e Zhao (20l3) corroboram o impacto da revisão das taxas de desconto e acrescentam o impacto relevante da reavaliação dos fluxos de caixa futuros das organizações, que costumam ser revisados - reduzidos - em momentos de crise.

Nesse sentido, Giot (2005) examina a relação inversamente proporcional entre o índice de volatilidade e o índice da Bolsa. $O$ autor observa que momentos de baixa no mercado acionário são acompanhados por altos índices de volatilidade, porém, essa relação se torna mais fraca quando ocorre um aumento no retorno das ações, não sendo estes acompanhados por valores muito baixos nos índices de volatilidade. Essas evidências revelam uma assimetria entre as variáveis estudadas.

Essa relação entre o VIX e o Standard \& Poor's 500 é amplamente estudada. Isso porque os índices de volatilidade possibilitam a negociação de derivativos sobre o nível de incerteza futura, permitindo assim o gerenciamento do risco de uma carteira de opções, da mesma maneira que se gerencia o risco de taxa de juros em uma carteira de renda fixa (DARIO, 2007). Esse gerenciamento pode ser feito através de outros mecanismos do mercado, porém são mais custosos e ineficientes (DARIO, 2007;WOO;VICENTE; BARBEDO, 2009). Nesse sentido, é importante que o $\mathrm{FXvol}$ se consolide como uma referência de mercado, possibilitando uma futura aplicação da sua metodologia ao índice da bolsa brasileira, assim como comentado por Dario (2007).

\section{Índice de Volatilidade Cambial Brasileiro}

O Índice de Volatilidade Cambial Brasileiro foi apresentado por Dario (2007). Seu trabalho propõe que o FXvol siga os moldes do VIX, baseado no estudo de Demeterfi, Derman, Kamal et al. (1999), que propuseram a utilização de um portfólio replicante para o apreçamento de swaps de variância. O FXvol indica a volatilidade implícita da taxa de câmbio BRL/ USD para os próximos 21 dias úteis, utilizando-se do próximo vencimento das opções, caso a data coincida com os 21 dias ou seja realizada uma interpolação entre os dois próximos vencimentos (DARIO, 2007).

Dario (2007) utiliza da seguinte forma para calcular o valor do swap de variância para o mercado brasileiro.

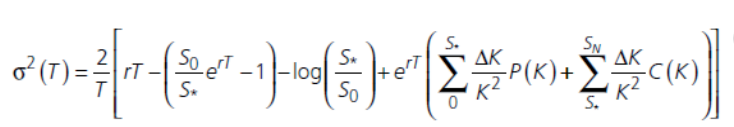

Onde:

- T - prazo do contrato;

- $r$ - taxa de juros para o prazo T;

- $\quad$ SO - preço do ativo em t zero;

- $S^{*}$ - parâmetro utilizado para replicar o payoff do contrato replicante, funciona como o limiar de escolha entre as opções de compra e venda;

- Sn - último preço de exercício disponível para a opção;

- K - preço de exercício;

- $\mathrm{P}(\mathrm{K})$ - preço livre de arbitragem da opção de venda com preço de exercício K;

- $\mathrm{C}(\mathrm{K})$ - preço livre de arbitragem da opção de compra com preço de exercício K.

Em seguida, é necessário interpolar os valores encontrados para os dois próximos vencimentos, se o próximo vencimento não coincidir com 21 dias úteis. Nesse 
caso, a fórmula apresentada a seguir é empregada, segundo Dario (2007).

$$
F X v o l=\sqrt{\left(T \sigma^{2}(T)\left[\frac{D U_{T 1}-D U_{21}}{D U_{T 1}-D U_{T}}\right]+T_{1} \sigma^{2}\left(T_{1}\right)\left[\frac{D U_{21}-D U_{T}}{D U_{T 1}-D U_{T}}\right]\right) \times \frac{D U_{252}}{D U_{21}}}(2)
$$

Apesar de o FXvol ter sido apresentado por Dario (2007) e incorporado aos índices da BM\&FBovespa em 2007, o autor reconstruiu a série até outubro de 2005 e evidenciou a correlação moderada entre o FXvol e o dólar spot e o índice lbovespa.

A consolidação do FXvol como um benchmark de mercado e posterior criação de derivativos sobre esse índice permitiram um controle maior por parte dos agentes do mercado do risco envolvido nas variações da taxa de câmbio. Isso porque o aumento da volatilidade cambial acarreta diversos efeitos negativos sobre o Brasil, como, por exemplo, a redução do comércio com seus parceiros internacionais, devido ao aumento do risco cambial. Bittencourt, Larson e Thompson (2007) evidenciaram o efeito negativo da volatilidade cambial no comércio brasileiro com os demais países que compõem o Mercosul.

Este efeito é reforçado por Barros (2009), ao verificar que o período de maior troca de mercado, entre Brasil e Argentina, se deu no momento de maior estabilidade cambial de ambas as moedas. Pode-se então constatar que um aumento da incerteza sobre o valor futuro a ser recebido (pago) pela venda (compra) de mercadorias desestimula a economia brasileira.

Poder-se-ia argumentar que os agentes de mercado têm a possibilidade de se proteger dessas variações no mercado de derivativos. Entretanto, como já foi exposto anteriormente, não é possível replicar de maneira robusta a volatilidade da taxa de câmbio utilizando-se de instrumentos financeiros presentes no mercado. Perde-se, assim, um mecanismo importante na proteção contra as variações cambiais.

Além do impacto exposto, variações inesperadas na taxa de câmbio podem gerar choques no mercado acionário, como aponta Grôppo (2006), ao analisar que uma depreciação adversa do real na ordem de $10 \%$ geraria uma queda no índice lbovespa de II,6\%. A partir dessas questões é que a pesquisa foi motivada, pelo interesse de se compreender a relação contemporânea entre o FXvol, a Ptax e o lbovespa. Para tanto, tomou-se por base o estudo de Giot (2005), apresentado a seguir, no qual o autor estudou a relação de dois índices da bolsa e seus respectivos índices de volatilidade.

\section{Modelo de Giot}

Em seu trabalho, Giot (2005) buscou evidenciar a existência de uma relação entre os retornos futuros da bolsa de valores e seu índice de volatilidade. $O$ estudo focou em avaliar duas correlações: uma entre o S\&P 100 (utilizando-se do índice OEX) e o VIX; e a outra entre o Nasdaq 100 (NDX) e oVXN (seu indexador da volatilidade implícita).

Foram então criadas as seguintes variáveis a serem estudadas:

$$
\begin{aligned}
& \bullet \rightarrow R_{O E X, t}=\ln \left(O E X_{t}\right)-\ln \left(O E X_{t-1}\right) ; \\
& \bullet \rightarrow R_{N D X, t}=\ln \left(N D X_{t}\right)-\ln \left(N D X_{t-1}\right) ; \\
& \bullet \rightarrow R_{V I X, t}=\ln \left(V I X_{t}\right)-\ln \left(V I X_{t-1}\right) ; \\
& \bullet \rightarrow R_{V X N, t}=\ln \left(V X N_{t}\right)-\ln \left(V X N_{t-1}\right) .
\end{aligned}
$$

Onde o retorno $(R)$ do índice, seja da bolsa de valores ou da volatilidade, é a diferença entre os logaritmos neperianos do valor do índice em $\mathrm{t}$ e $\mathrm{t}$ - I.

Em seguida, o autor utiliza-se das seguin- 
tes regressões múltiplas para avaliar a existência de relação pertinente:

$$
\begin{aligned}
& R_{V I X, t}=\beta_{0}^{+} D_{t}^{+}+\beta_{0}^{-} D_{t}^{-}+\beta_{1}^{+} R_{O E X, t} D_{t}^{+}+\beta_{1}^{-} R_{O E X, t} D_{t}^{-}+\varepsilon_{t} \rightarrow \text { (3) } \\
& R_{V X N, t}=\beta_{0}^{+} D_{t}^{+}+\beta_{0}^{-} D_{t}^{-}+\beta_{1}^{+} R_{N D X, t} D_{t}^{+}+\beta_{1}^{-} R_{N D X, t} D_{t}^{-}+\varepsilon_{t} \rightarrow \text { (4) }
\end{aligned}
$$

Sendo uma variável binária, zero um, que adquire valor zero (um) quando o retorno for positivo (negativo) e. Dessa forma se anulará o terceiro (quarto) termo da equação, caso o retorno seja positivo (negativo).

A partir dessas equações, o autor pretendeu avaliar se haveria uma relação entre os retornos positivos ou negativos da bolsa e o índice de volatilidade implícita. Os resultados obtidos apontaram para uma relação estatisticamente relevante entre ambos os retornos; entretanto, os retornos negativos demonstraram maior impacto, i.e., tem valor absoluto superior a .

O autor ainda realizou teste estatístico para verificar se a diferença entre os valores era estatisticamente relevante, pois o trabalho foi elaborado com diversos cortes amostrais ao longo do tempo, e o resultado apontou para a relevância da diferença, constatando assim a assimetria de impacto entre os retornos negativo e positivo.

Após apontar essa correlação linear, Giot (2005) acrescentou duas variáveis ao modelo empregado anteriormente, com o intuito de verificar se o quadrado dos retornos poderia conter alguma informação adicional. Os valores obtidos para o retorno quadrático não se mostraram estatisticamente relevantes. Além disso, o poder de explicação do primeiro modelo para o segundo não foi alterado, fato que deixou clara a não correlação entre os retornos quadráticos e o índice de volatilidade implícita.

Em uma segunda fase do estudo, Giot (2005) implementa uma metodologia com intuito de verificar se o índice de volatilidade tem a capacidade de funcionar como um indicador antecedente, ou seja, se observações muito altas (baixas) de expectativa futura para a volatilidade correspondem a retornos futuros do mercado mais altos (baixos). A ideia por trás do teste é que períodos de volatilidade alta indicam incerteza no mercado, o que leva os agentes a aumentarem a taxa de desconto do fluxo de retorno dos ativos, gerando queda nos preços. Essa situação seria uma oportunidade para entrada em posições long.

O autor fixa então uma janela móvel de dois anos e verifica se, em um dado instante de tempo t, o valor observado do índice está relativamente alto ou baixo em relação aos valores da janela.Armazenada essa informação, é verificado o retorno do mercado em um horizonte futuro de tempo. Os resultados indicam uma boa capacidade preditiva do índice de volatilidade.

\section{Base de Dados e Metodologia Dados utilizados}

Neste estudo, foram utilizadas as séries históricas diárias do FXvol, Ptax e o índice lbovespa, fornecidas pelo sistema de recuperação de dados da BM\&FBovespa. Apesar de o FXvol iniciar sua divulgação em julho de 2007, foi possível recuperar a série desde outubro de 2005, em razão de o Prof. Alan de Genaro Dário ter cedido, gentilmente, a série de dados reconstruída sinteticamente em seu trabalho. Sendo assim, as análises foram realizadas, considerando o período de outubro de 2005 até fevereiro de 2015.

\section{Análise Estatística e Análise das Relações Contemporâneas}

Inicialmente, foi feita uma análise pre- 
liminar dos dados a partir da matriz de correlação e visualização gráfica das séries históricas. Buscou-se, nesse primeiro momento, compreender de maneira preliminar a inter-relação das variáveis. Em um segundo momento, baseado no estudo de Giot (2005), calcularam-se as variações diárias de cada uma das variáveis a partir da equação 5 e foram realizadas as regressões múltiplas, utilizando-se das equações 6 e 7, expostas a seguir:

$$
\begin{array}{r}
\Delta X, t=\ln (X, t)-\ln (X, t-\mid) \\
\Delta_{F X v o l, t}=\beta_{0}^{+} D_{t}^{+}+\beta_{0}^{-} D_{t}^{-}+\beta_{1}^{+} \Delta_{\text {dolar }, t} D_{t}^{+}+\beta_{1}^{-} \Delta_{\text {doiar }, t} D_{t}^{-}+\varepsilon_{t} \rightarrow \\
\Delta_{F X v o l, t}=\beta_{0}^{+} D_{t}^{+}+\beta_{0}^{-} D_{t}^{-}+\beta_{1}^{+} \Delta_{\text {lbov }, t} D_{t}^{+}+\beta_{1}^{-} \Delta_{\text {lbov }, t} D_{t}^{-}+\varepsilon_{t} \rightarrow \rightarrow
\end{array}
$$

Assim como no trabalho de Giot (2005), é uma variável binária que adquire valor zero (um) quando o retorno for positivo (negativo) e .Dessa forma, se anulará o terceiro (quarto) termo da equação, caso o retorno seja positivo (negativo).

A modelagem sugere a influência do dólar e do lbov sobre o FXvol, captando a relação entre as variáveis, sem entretanto fixar o sinal da influência de uma variável sobre a outra. Esse direcionamento fica a cargo da literatura e dos estudos pregressos. Nesse caso, como foi exposto no referencial teórico, espera-se uma influência negativa do FXvol sobre o lbov e uma inter-relação com o dólar significativa, visto o FXvol ser calculado a partir de derivativos de taxa de câmbio.

As regressões descritas nas equações (5) - (7) foram calculadas, em um primeiro momento, com toda a série histórica e os resultados analisados. A seguir, procurou-se avaliar se o cenário de maior incerteza influencia a relação entre as variáveis em estudo, a série foi então dividida em três momentos:

- Momento I: outubro de 2005 até julho de 2008;
- Momento 2: setembro de 2008 até dezembro de 2009;

- Momento 3: janeiro de 2010 até fevereiro de 2015 .

\section{Análise do FXvol como Indicador Antecedente}

A metodologia exposta anteriormente focou investigar a relação contemporânea entre o FXvol, a Ptax e o lbovespa. Com o intuito de avaliar a capacidade do FXvol de captar a possível relação entre o nível de incerteza presente no mercado e os retornos futuros da taxa de câmbio e do índice de ações, aplicou-se uma metodologia adaptada de Giot (2005). Em um dado momento t, observou-se o FXvol e o retorno futuro do lbovespa e do dólar para os próximos I, 10,2I e 63 dias úteis. Classificaram-se os valores do FXvol observados em t, considerando-se a divisão da série histórica do FXvol até o momento t-I em vinte percentis. Considerou-se que os cinco primeiros percentis caracterizavam um baixo nível de volatilidade; os percentis seis a quinze, um nível médio; e do décimo sexto percentil em diante, uma volatilidade alta.

Repetido esse processo de um dado momento $t$ até o final da série, classificouse a volatilidade de cada instante de tempo como: baixa, média ou alta. Compararamse então os retornos médios futuros das classes de baixa e alta volatilidade e, assim, pôde-se avaliar a relação entre o nível de incerteza presente no mercado e as variações futuras do dólar e do lbovespa.

\section{Resultados}

$\mathrm{O}$ índice $\mathrm{FXvol}$, registrado diariamente, foi examinado para o período de outubro de 2005 a fevereiro de 2015. A amostra contém 2.277 observações. Foram elabo- 
radas as análises descritivas, análise da assimetria na variação diária da série e as relações entre o FXvol e a Ptax e o lbovespa.

A Figura I mostra a evolução diária do logaritmo do índice $\mathrm{FXvol}$. $\mathrm{O}$ índice apresenta um comportamento bastante volátil, com uma oscilação entre setembro de 2008 e dezembro de 2009, período em que o mundo enfrentou $o$ início da crise econômica do subprime (apesar de a crise ter seu início em julho de 2007, seus impactos mais relevantes foram percebidos no período citado).

Nesse período, o índice, cuja média histórica é de aproximadamente 2,67, chegou a atingir valor superior a 4, 17. Outro período de alta ocorre no final de $201 \mathrm{I}$, possivelmente resultado do impacto causado pela rápida redução na taxa básica de juros da economia, a Taxa Selic. No restante do período, o índice se mantém em baixos níveis, embora bastante volátil.

A Figura 2 mostra o logaritmo das três séries históricas estudadas, de outubro de 2005 a fevereiro de 20I5. Nota-se a relação negativa presente entre o índice Bovespa e a Ptax, principalmente durante a crise de 2008 . Da mesma maneira, percebe-se que níveis mais altos do FXvol estão atrelados a quedas no índice da bolsa e a aumentos na Ptax.

A forte correlação negativa entre a Taxa de Câmbio Brasil-EUA (Ptax) e o índice do mercado de ações (Ibovespa), ilustrada na Figura 3 e na Tabela I, já foi amplamente documentada na literatura acadêmica, como, por exemplo, em Silva e Coronel (2012), Grôppo (2006) e Pimenta Júnior e Scherma (2005).

O FXvol representa a incerteza futura dos investidores em relação a taxa de câmbio. Essas duas evidências nos motivam a questionar se $\mathrm{FXvol}$ tem relação com o lbovespa e, mais, se seria um bom indicador antecedente para o comportamento do mercado de ações, visto que ele seria um termômetro da incerteza do investidor um período a frente.

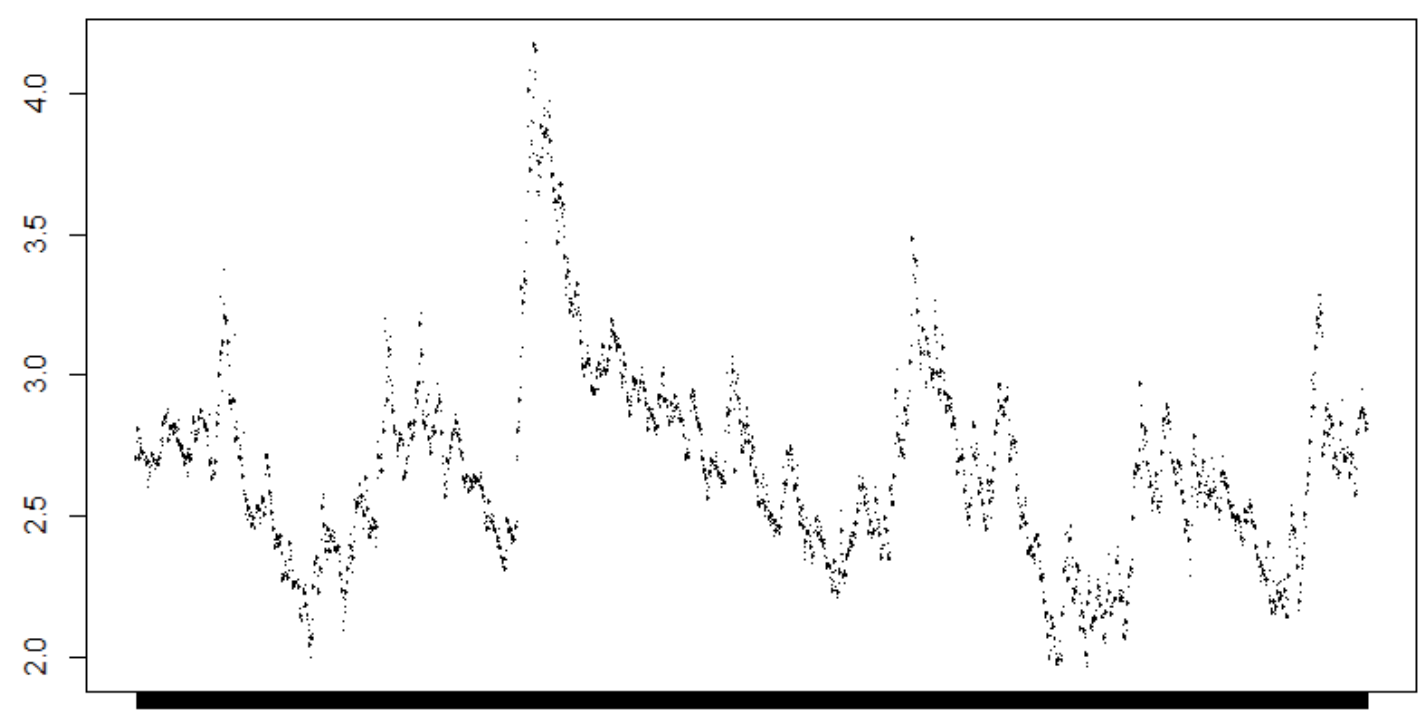

$2005-10-03 \quad 2007-01-18 \quad 2008-05-27 \quad 2009-10-02 \quad 2011-01-28 \quad 2012-05-15 \quad 2013-09-02 \quad 2014-12-11$

FIGURA 1 - Logaritmo do índice FXvol

Fonte: Dados coletados no sistema da BM\&FBovespa.

Nota: O valor do FXvol informado pela bolsa é anualizado. 


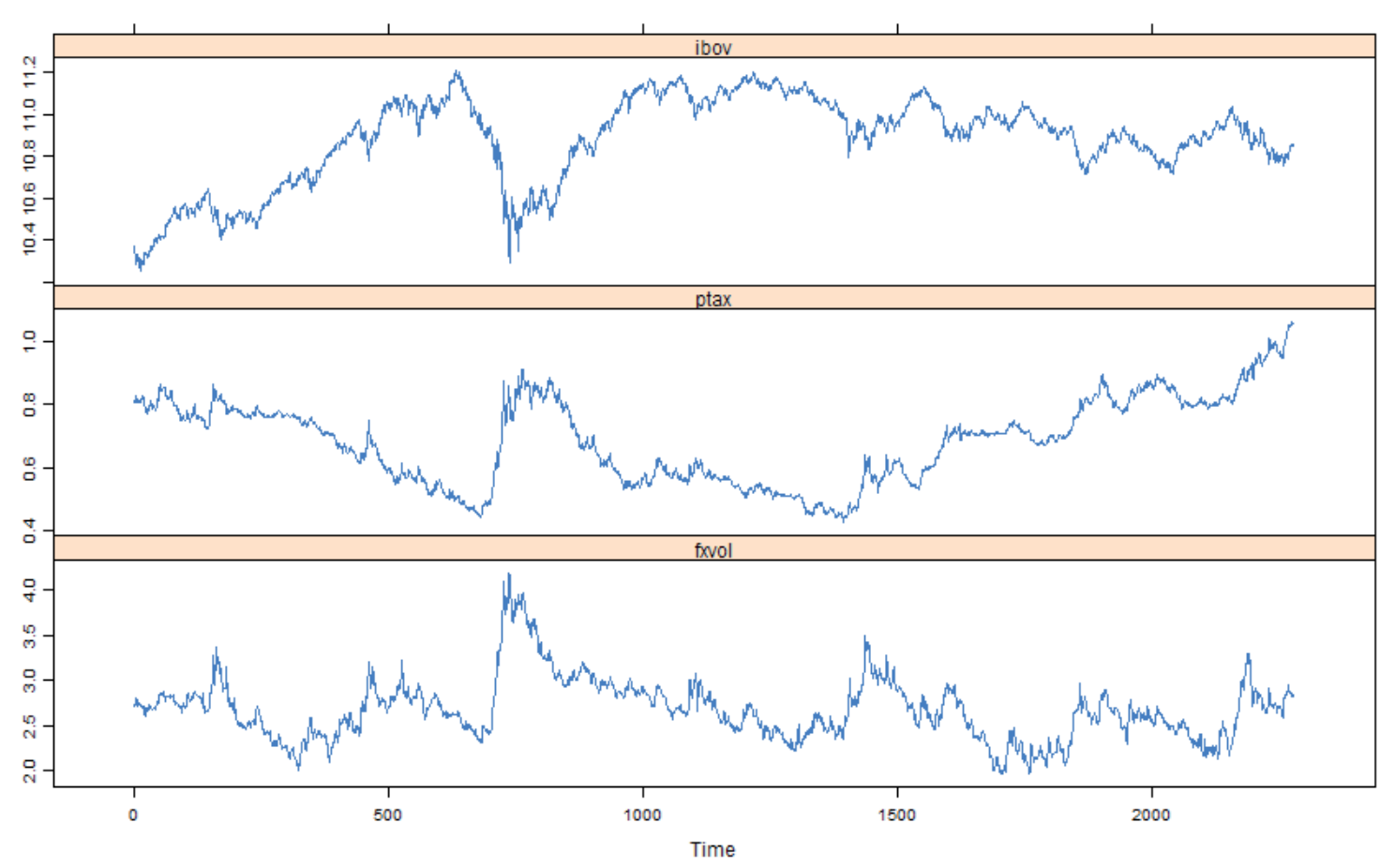

FIGURA 2 - Séries históricas do índice Bovespa, Ptax e FXvol logaritmizadas

Fonte: Dados coletados no sistema da BM\&FBovespa e na Bloomberg.

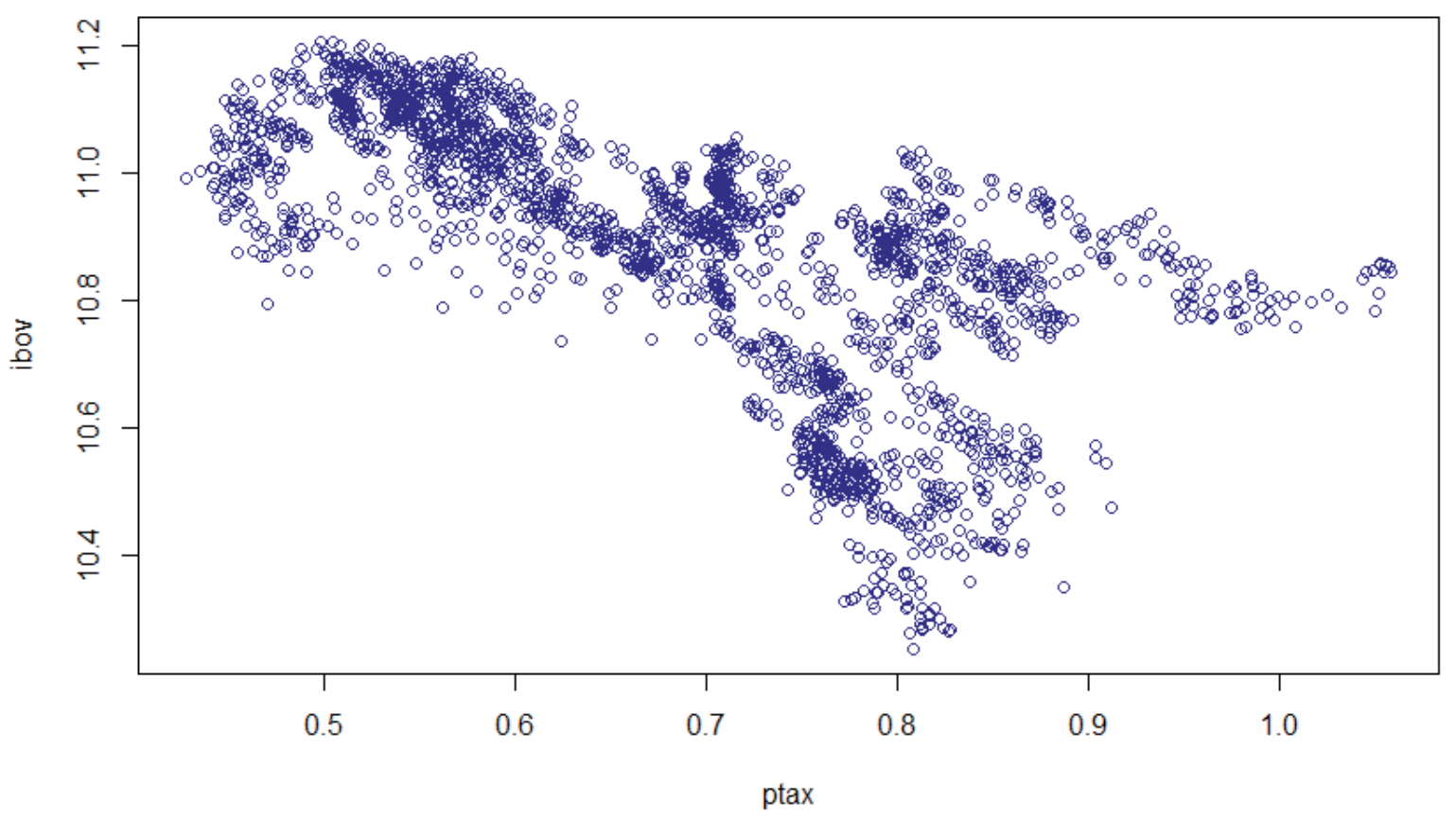

FIGURA 3 - Gráfico de dispersão relacionando o lbovespa e a Ptax

Fonte: Dados coletados na Bloomberg. 
TABELA 1 - Correlação de Pearson entre as variáveis

\begin{tabular}{cccc}
\hline & lbov & Ptax & FXvol \\
\hline Ibov & 1 & $-0,6745$ & $-0,2956$ \\
Ptax & $-0,6745$ & 1 & 0,1436 \\
FXvol & $-0,2956$ & 0,1436 & 1 \\
\hline
\end{tabular}

Fonte: Dados coletados no sistema da BM\&FBovespa e na Bloomberg.

$\mathrm{Na}$ Tabela 2, são apresentadas as propriedades estatísticas do índice FXvol.As mediadas tradicionais, a saber: a média, a variância, o desvio-padrão, o mínimo, o primeiro quartil, a mediana, o terceiro quartil, o máximo, a assimetria e a curtose para o log da série histórica do FXvol. Realizou-se também o teste de normalidade de Jarque-Bera.

$\mathrm{Na}$ primeira metade da amostra, há uma ligeira elevação na média e no desvio padrão do índice, bem como em sua assimetria, o que já era esperado em decorrência da crise econômica de 2008. Como é um índice que representa a incerteza do investidor, seria normal observar-se um aumento da assimetria à direta em períodos de maior instabilidade. Os dados não apresentam variações significativas entre os períodos, apesar do gráfico na Figura I aparentemente sugerir dois padrões distintos de comportamento para as duas metades da amostra. Além disso, o teste Jarque-Bera rejeita a hipótese nula de normalidade para a série histórica do índice de volatilidade cambial.

Antes de seguir, fez-se necessário verificar se as séries eram estacionárias ou não, através do Teste Augmented Dickey-Fuller. A importância do teste se dá pelo fato de as séries não estacionárias, por terem uma tendência temporal, poderem apresentar alto poder explicativo, mesmo que as variáveis não fossem correlacionadas (BROOKS, 20I4).

Os resultados dos testes são expostos na Tabela 3. As três séries não apresentam raiz unitária, ou seja, são estacionárias. No-

TABELA 2 - Estatística descritiva para o logaritmo do índice FXvol

\begin{tabular}{l|c|c|c}
\hline \multirow{2}{*}{ Estatística } & \multicolumn{2}{c}{ Amostra } \\
\cline { 2 - 4 } & Primeira Metade & Segunda Metade & Completa \\
\hline Máximo & 4,1724 & 3,4836 & 4,1724 \\
\hline Mínimo & 2,0030 & 1,9691 & 1,9691 \\
\hline $1^{\circ}$ Quartil & 2,5663 & 2,3631 & 2,4485 \\
\hline $3^{\circ}$ Quartil & 2,9331 & 2,7168 & 2,8496 \\
\hline Média & 2,7933 & 2,5532 & 2,6732 \\
\hline Mediana & 2,7763 & 2,5417 & 2,6516 \\
\hline Variância & 0,1296 & 0,0727 & 0,1155 \\
\hline Desvio-padrão & 0,3600 & 0,2697 & 0,3399 \\
\hline Assimetria & 1,0278 & 0,3813 & 0,9664 \\
\hline Curtose & 4,8115 & 3,1434 & 5,0486 \\
\hline Jarque-Bera & $<0.001$ & $<0.001$ & $<0.001$ \\
\hline A tabela tratas
\end{tabular}

A tabela trata das propriedades estatísticas do logaritmo da série do FXvol, sendo a primeira metade da série compreendendo o período de outubro de 2005 a 14 de julho de 2010, enquanto a segunda metade de 15 de julho de 2010 a fevereiro de 2015. 
TABELA 3 - Teste de Estacionariedade

\begin{tabular}{l|c|c}
\hline \multicolumn{1}{c|}{ Retornos } & Augmented Dickey-Fuller & p-valor \\
\hline FXvol & $-11,9966$ & $<0.01$ \\
\hline Ibovespa & $-13,0245$ & $<0.01$ \\
\hline Ptax & $-11,6614$ & $<0.01$ \\
\hline
\end{tabular}

Fonte: Dados coletados no sistema da BM\&FBovespa e na Bloomberg.

A hipótese nula do teste é de que a série apresenta raiz unitária e a hipótese alternativa de que a série não tem raiz unitária, sendo assim estacionária. O teste foi realizado com 13 lags.

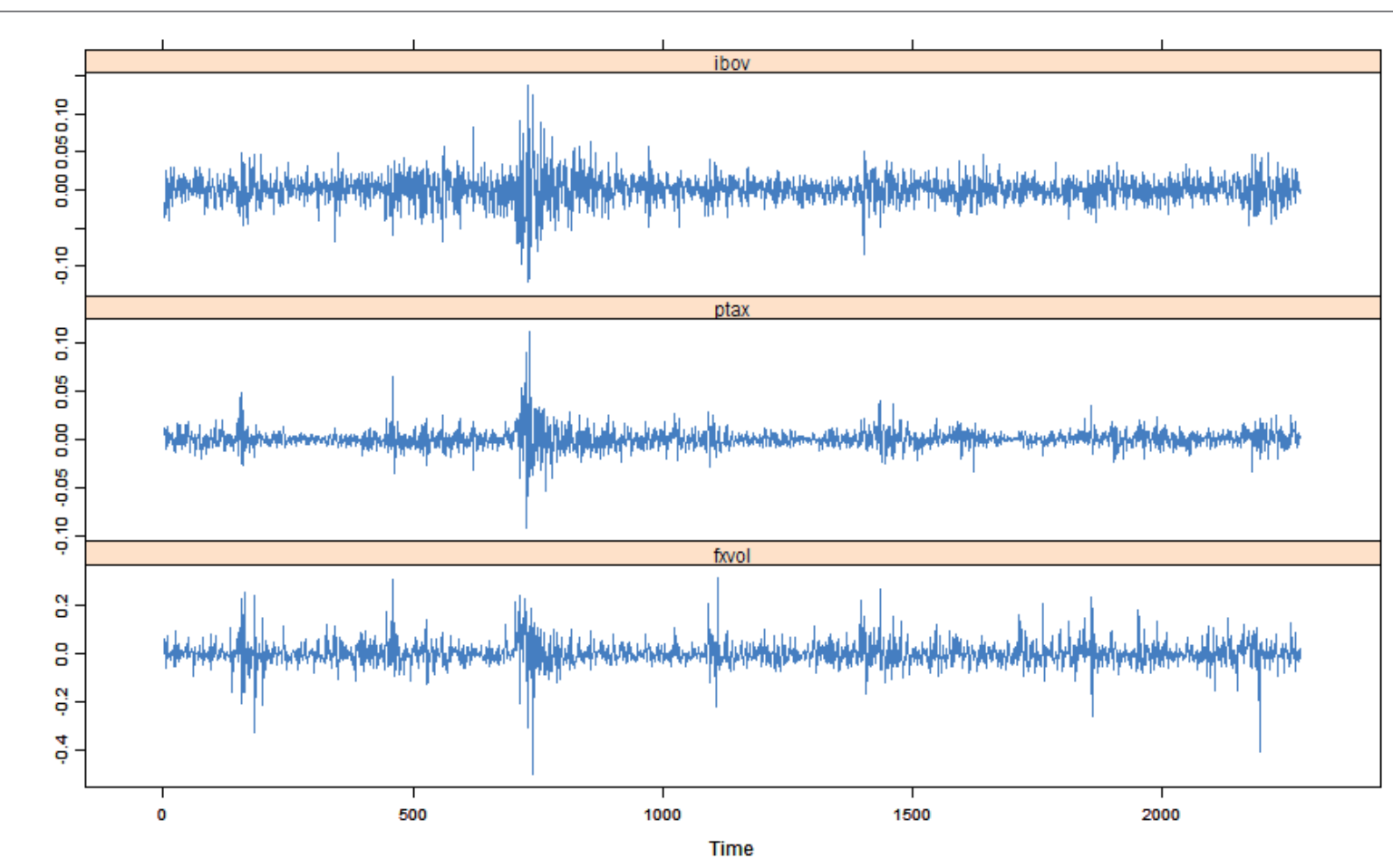

FIGURA 4 - Séries históricas do retorno diário do índice Bovespa, Ptax e FXvol

Fonte: Dados coletados no sistema da BM\&FBovespa e na Bloomberg.

ta-se o mesmo na Figura 4, que contém o retorno diário das três séries.

\section{Relação entre o FXvol e o lbovespa}

Em um primeiro momento, a regressão foi calculada para a série como um todo. Em seguida, procurou-se avaliar se o cenário de crise influencia a relação entre as variáveis, Sendo assim, a série foi dividida em três períodos:

- Momento I: outubro de 2005 até julho de 2008;

- Momento 2: setembro de 2008 até dezembro de 2009;
- Momento 3: janeiro de 2010 até fevereiro de 2015.

$\mathrm{Na}$ tabela a seguir, são apresentados os resultados da regressão para o índice Ibovespa, com a série histórica completa.

Os resultados da regressão indicam que o retorno negativo do mercado acionário está associado com um maior impacto no índice de volatilidade cambial que os retornos positivos. Um aumento de $1 \%$ no índice acionário reduz o $\mathrm{FXvol}$ em $0,68 \%$, enquanto uma redução de $1 \%$ gera um aumento de $1,09 \%$. 
TABELA 4 - Regressão Completa para o lbovespa

\begin{tabular}{c|c|c|c|c}
\hline \multicolumn{5}{c}{ Regressão: $\Delta_{F X v o l, t}=\beta_{0}^{+} D_{t}^{+}+\beta_{0}^{-} D_{t}^{-}+\beta_{1}^{+} \Delta_{\text {Ibov }, t} D_{t}^{+}+\beta_{1}^{-} \Delta_{\text {Ibov,t }} D_{t}^{-}+\varepsilon_{t}$} \\
\hline Variáveis & Coeficiente & Erro-padrão & Estatística t & p-valor \\
\hline$D_{t}^{+}$ & 0,0001 & 0,0030 & 0,0439 & 0,9649 \\
\hline$D_{t}^{-}$ & $-0,0051$ & 0,0019 & $-2,6439$ & $0.0083^{* \star *}$ \\
\hline$\Delta_{\text {Ibov }, t} D_{t}^{+}$ & $-0,6842$ & 0,2537 & $-2,6969$ & $0.0071^{* \star *}$ \\
\hline$\Delta_{\text {Ibov }, t} D_{t}^{-}$ & $-1,0923$ & 0,1418 & $-7,7006$ & $2.006 \mathrm{e}-14^{* \star *}$ \\
\hline
\end{tabular}

Fonte: Dados coletados no sistema da BM\&FBovespa e na Bloomberg.Nota: O teste F teve $p$-valor < 2.2e-16 e Breusch-Pagan de 4.296e-16, por isso os estimadores foram corrigidos usando a matriz de covariância de White.

Significância: ${ }^{* * *},{ }^{* *} \mathrm{e}{ }^{*}$ correspondem a $0.01,0.05$ e 0.1 , respectivamente.

Como era esperado, retornos positivos do lbovespa estão associados com redução do FXvol, enquanto que retornos negativos estão associados a aumentos no FXvol, refletindo a relação próxima entre mercado acionário e cambial no Brasil. Além disso, diferente do que ocorre no mercado americano exposto por Giot (2005), os retornos negativos sofrem um amortecimento de $0,005 \%$.Apesar de estatisticamente diferente de zero, esse valor é muito pequeno, mostrando-se assim de pouca influência.

A Tabela 5 apresenta o resultado das regressões para os momentos I a 3 .

Os resultados apontam para uma insensibilidade do FXvol ao retorno positivo, a partir da crise de 2008. Nota-se que, no momento I, os retornos tinham um impacto assimétrico, ainda que pequeno, e, após a crise, apenas a associação entre o retorno negativo e o aumento da volatilidade persistiu em intensidade menor, momentos 2 e 3.

Esse fato sugere uma mudança na realidade do mercado acionário, que vem desde a passagem dos piores efeitos da crise, com um comportamento de "caranguejo”, andando para o lado. Nesse contexto, apenas as notícias ruins têm importância, desconsiderando-se o impacto das notícias boas, seja por falta das mesmas, seja por insensibilidade, devido ao momento vivido. De maneira geral, os resultados mostraram que o FXvol age como um captador do medo do mercado, pois retornos negativos do lbovespa estão atrelados a um aumento no índice de volatilidade cambial.

\section{Relação entre o FXvol e a Ptax}

O mesmo procedimento aplicado ao Ibovespa foi aplicado à Ptax, sendo exposto na Tabela 6 o resultado da regressão realizada para toda a série histórica.

Os resultados da regressão apontam para uma associação entre valorização do dólar e o aumento da volatilidade, assim como associação entre desvalorização do dólar e redução da volatilidade. $\bigcirc$ resultado era esperado, pois a valorização da moeda americana evidencia uma piora no cenário nacional, dado que a taxa de câmbio reflete a desproporcionalidade entre as economias. Nota-se a assimetria entre a valorização e desvalorização da moeda americana, mostrando que a piora no cenário nacional impacta o índice de volatilidade em quase três vezes mais do que a melhora. Assim como ocorreu com o lbovespa, o retorno negativo da Ptax sofre um amortecimento que, apesar de estatisticamente relevante, mostrou-se de pouca influência.

Seguiu-se a análise da regressão para os três momentos estudados.

A Tabela 7 mostra os resultados das últimas três regressões calculadas. Nota-se 
TABELA 5 - Regressão para o lbovespa considerando momento de análise

\begin{tabular}{|c|c|c|c|c|}
\hline \multicolumn{5}{|c|}{ Momento 1} \\
\hline Variáveis & Coeficiente & Erro-padrão & Estatística t & p-valor \\
\hline$D_{t}^{+}$ & 0,0037 & 0,0041 & 0,8949 & 0,3711 \\
\hline$D_{t}^{-}$ & $-0,0061$ & 0,0039 & $-1,5712$ & 0,1166 \\
\hline$\Delta_{\text {Ibov }, t} D_{t}^{+}$ & $-0,9892$ & 0,2852 & $-3,4686$ & $0.0006^{\star \star *}$ \\
\hline$\Delta_{I b o v, t} D_{t}^{-}$ & $-1,1762$ & 0,2971 & $-3,9587$ & $8.31 \mathrm{e}-05^{\star \star *}$ \\
\hline \multicolumn{5}{|c|}{ Momento 2} \\
\hline Variáveis & Coeficiente & Erro-padrão & Estatística t & p-valor \\
\hline$D_{t}^{+}$ & 0,0021 & 0,0075 & 0,2767 & 0,7822 \\
\hline$D_{t}^{-}$ & $-0,0057$ & 0,0045 & $-1,2579$ & 0,2094 \\
\hline$\Delta_{\text {Ibov }, t} D_{t}^{+}$ & $-0,7526$ & 0,4976 & $-1,5124$ & 0,1315 \\
\hline$\Delta_{\text {Ibov }, t} D_{t}^{-}$ & $-1,0874$ & 0,2051 & $-5,3012$ & $2.211 \mathrm{e}-07^{\star \star \star}$ \\
\hline \multicolumn{5}{|c|}{ Momento 3} \\
\hline Variáveis & Coeficiente & Erro-padrão & Estatística t & p-valor \\
\hline$D_{t}^{+}$ & $-0,0037$ & 0,0033 & $-1,1295$ & 0,2589 \\
\hline$D_{t}^{-}$ & $-0,0045$ & 0,0031 & $-1,4270$ & 0,1538 \\
\hline$\Delta_{\text {Ibov }, t} D_{t}^{+}$ & $-0,3191$ & 0,2793 & $-1,1424$ & 0,2535 \\
\hline$\Delta_{\text {Ibov }, t} D_{t}^{-}$ & $-1,0416$ & 0,3232 & $-3,2225$ & $0.0013^{\star \star *}$ \\
\hline
\end{tabular}

Fonte: Dados coletados no sistema da BM\&FBovespa e na Bloomberg.

Nota: Os momentos de 1 a 3 apresentaram p-valor no teste $F$ de $<2.2 \mathrm{e}-16,4.52 \mathrm{e}-13$ e $7.341 \mathrm{e}-12$, respectivamente. Os momentos 1 e 2 apresentaram p-valor no teste de Breusch-Pagan de 0.04587 e 2.448 e- 07 , respectivamente, e por isso os estimadores foram corrigidos usando a matriz de covariância de White. O momento 3, além de apresentar heterocedasticidade dos resíduos, p-valor do teste de Breusch-Pagan de 5.049e-05, também apresentou auto correlação entre os resíduos, p-valor do teste de Durbin-Watson de 9.938e-05, e por isso os estimadores foram corrigidos usando a matriz de covariância de Newey-West (matriz HAC).

A amostra Antes da Crise conteve 700 observações, a amostra da Crise 309 observações e a Pós Crise 1267 observações totalizando 2276 observações. Perdeu-se uma observação da amostra inicial para o cálculo do retorno.

Significância: ${ }^{* * *},{ }^{* *} \mathrm{e}^{*}$ correspondem a $0.01,0.05$ e 0.1 , respectivamente.

TABELA 6 - Regressão Completa para a Ptax

Regressão: $\Delta_{F X v o l, t}=\beta_{0}^{+} D_{t}^{+}+\beta_{0}^{-} D_{t}^{-}+\beta_{1}^{+} \Delta_{p \operatorname{tax}, t} D_{t}^{+}+\beta_{1}^{-} \Delta_{p t a x, t} D_{t}^{-}+\varepsilon_{t}$

\begin{tabular}{c|c|c|c|c}
\hline Variáveis & Coeficiente & Erro-padrão & Estatística t & p-valor \\
\hline$D_{t}^{+}$ & $-0,0022$ & 0,0028 & $-0,8013$ & 0,4231 \\
\hline$D_{t}^{-}$ & $-0,0100$ & 0,0024 & $-4,2384$ & $2.341 \mathrm{e}-05^{\star * *}$ \\
\hline$\Delta_{\text {Ibov,t}} D_{t}^{+}$ & 2,9297 & 0,4543 & 6,4495 & $1.367 \mathrm{e}-10^{\text {***}}$ \\
\hline$\Delta_{\text {Ibov,t }} D_{t}^{-}$ & 1,0230 & 0,4046 & 2,5288 & $0.01151^{\text {** }}$ \\
\hline
\end{tabular}

Fonte: Dados coletados no sistema da BM\&FBovespa e na Bloomberg.

Nota: $O$ teste $F$ teve p-valor $<2.2 \mathrm{e}-16$ e Breusch-Pagan de $6.358 \mathrm{e}-14$, por isso os estimadores foram corrigidos usando a matriz de covariância de White.

Significância: ${ }^{* * *},{ }^{* *} \mathrm{e}^{*}$ correspondem a 0.01, 0.05 e 0.1, respectivamente.

que o aumento da volatilidade, associado à valorização do dólar, é menor no período de alta volatilidade, momento 2 , em relação aos períodos de baixa volatilidade, mo- mentos I e 2. Uma possível explicação para esse fenômeno é o fato de a volatilidade ter atingido patamar muito elevado, não sendo mais tão impactada pela valorização do 


\section{TABELA 7 - Regressão para a Ptax considerando momento de análise}

\begin{tabular}{|c|c|c|c|c|}
\hline \multicolumn{5}{|c|}{ Momento 1} \\
\hline Variáveis & Coeficiente & Erro-padrão & Estatística t & p-valor \\
\hline$D_{t}^{+}$ & $-0,0087$ & 0,0033 & $-2,6514$ & $0.0082^{* * *}$ \\
\hline$D_{t}^{-}$ & $-0,0086$ & 0,0036 & $-2,3576$ & $0.0187^{* *}$ \\
\hline$\Delta_{\text {Ibov }, t} D_{t}^{+}$ & 3,9778 & 0,4559 & 8,7253 & $2.2 \mathrm{e}-16^{\star * *}$ \\
\hline$\Delta_{\text {Ibov }, t} D_{t}^{-}$ & 0,7055 & 0,7345 & 0,9605 & 0,3372 \\
\hline \multicolumn{5}{|c|}{ Momento 2} \\
\hline Variáveis & Coeficiente & Erro-padrão & Estatística t & p-valor \\
\hline$D_{t}^{+}$ & 0,0039 & 0,0057 & 0,6710 & 0,5024 \\
\hline$D_{t}^{-}$ & $-0,0050$ & 0,0057 & $-0,8620$ & 0,3891 \\
\hline$\Delta_{\text {Ibov }, t} D_{t}^{+}$ & 1,6693 & 0,2970 & 5,6200 & $4.31 \mathrm{e}-08^{\star * *}$ \\
\hline$\Delta_{\text {Ibov }, t} D_{t}^{-}$ & 1,3525 & 0,3617 & 3,7390 & $0.0002^{* * *}$ \\
\hline \multicolumn{5}{|c|}{ Momento 3} \\
\hline Variáveis & Coeficiente & Erro-padrão & Estatística t & p-valor \\
\hline$D_{t}^{+}$ & $-0,0098$ & 0,0027 & $-3,6197$ & $0.0003^{\star * *}$ \\
\hline$D_{t}^{-}$ & $-0,0131$ & 0,0020 & $-6,5768$ & $7.021 \mathrm{e}-11^{* * *}$ \\
\hline$\Delta_{\text {Ibov }, t} D_{t}^{+}$ & 4,6924 & 0,6105 & 7,6866 & $3.021 \mathrm{e}-14^{* * *}$ \\
\hline$\Delta_{\text {Ibov }, t} D_{t}^{-}$ & 0,8753 & 0,4127 & 2,1209 & $0.0341^{* *}$ \\
\hline
\end{tabular}

Fonte: Dados coletados no sistema da BM\&FBovespa e na Bloomberg.

Nota: Os momentos de 1 a 3 apresentaram p-valor no teste $\mathrm{F}$ de $<2.2 \mathrm{e}-16$. O momento 1 apresentou p-valor no teste de Breusch -Pagan de 0.0007536 e por isso os estimadores foram corrigidos usando a matriz de covariância de White. O momento 3, além de apresentar heterocedasticidade dos resíduos, p-valor do teste de Breusch-Pagan de 4.929e-06, também apresentou auto correlação entre os resíduos, p-valor do teste de Durbin-Watson de 0.008402 , e por isso os estimadores foram corrigidos usando a matriz de covariância de Newey-West (matriz HAC).

A amostra Antes da Crise conteve 700 observações, a amostra da Crise 309 observações e a Pós Crise 1267 observações totalizando 2276 observações. Perdeu-se uma observação da amostra inicial para o cálculo do retorno.

Significância: ${ }^{* *},{ }^{* *} \mathrm{e}^{*}$ correspondem a $0.01,0.05$ e 0.1 , respectivamente.

dólar devido à impossibilidade de aumento contínuo. Nesse momento, o impacto tanto da valorização como da desvalorização da moeda americana se aproximaram.

Importante notar a mudança no comportamento do $\mathrm{FXvol}$ frente às variações na Ptax antes e depois da crise. Enquanto antes, momento I, uma desvalorização da moeda americana causava uma pequena redução no índice de volatilidade. Após a crise, momento 3 , esse comportamento se alterou e o impacto passou a ser de 0,87\% para cada I\% de desvalorização. Além desse impacto, o fator de amortecimento do impacto de uma valorização sofre um aumento, assim como um reforço do movimento de redução do FXvol frente a uma desvalorização do dólar de $0,013 \%$, independente da magnitude da desvalorização.

Os resultados, como um todo, evidenciaram a ação do FXvol como um captador das variações do mercado cambial brasileiro, devido aos retornos positivos da Ptax estarem atrelados a fortes aumentos no índice de volatilidade cambial.

\section{FXvol como Indicador Antecedente}

Para investigar a capacidade preditiva do FXvol em relação ao retorno da Ptax 
e ao retorno do lbovespa, foram fixadas 252 observações "conhecidas" das variáveis em um tempo $\mathrm{t}=0$, e ordenou-se o FXvol (em logaritmo) em 20 percentis. Ao observar-se a ocorrência em $t=1$ classificou-se o FXvol no percentil correspondente e armazenaram-se os log -retornos da Ptax e do lbovespa para I dia, duas semanas, um mês e três meses à frente. Incorporou-se a nova observação $(t=l)$ ao histórico das observações conhecidas, fez-se um novo ordenamento em percentis e repetiu-se o procedimento para as observações em $t=t+l$, até o fim da amostra.

Ao final do processo, tomou-se a média dos log-retornos de Ptax e lbovespa relativos aos cinco primeiros percentis do FXvol - períodos de volatilidade bai$\mathrm{xa}$ - e dos cinco últimos - períodos de volatilidade alta. Assim obteve-se a média de retorno para cada prazo estudado seguinte a uma observação de baixa e de alta volatilidade.

Esse procedimento é semelhante ao descrito em Giot (2005). Porém, naquele artigo, o autor arbitra uma janela móvel de 2 anos para os dados observados, enquanto nesse trabalhou-se com toda a série histórica. A motivação da alteração no procedimento é que, além da escolha do prazo ter sido arbitrária, para um período longo de alteração do nível da volatilidade, como durante a crise de 2008, por exemplo, o nível alterado de volatilidade distorcerá a classificação em relação à série histórica. Uma volatilidade considerada baixa em um período de média de volatilidade alta pode ser alta em relação à série histórica.

O objetivo do trabalho é verificar se os retornos futuros, em períodos de volatilidade baixa, se comportam de for- ma diferente dos retornos em períodos de volatilidade alta. Logo, a utilização da série histórica total disponível pareceu mais adequada do que a fixação de uma janela.A média por quintil do $\mathrm{FX}$ vol pode ser vista na Figura 5.

Os resultados apresentados na Tabela 12 mostram que, em média, observações de baixa volatilidade são seguidas de retornos negativos na Ptax e positivos no lbovespa, para os prazos de duas semanas, um mês e três meses. Já os retornos seguintes a observações de alta volatilidade são, em média, positivos para a Ptax e negativos para o lbovespa. Para os retornos de I dia à frente, tanto a baixa volatilidade observada quanto a alta volatilidade observada são pouco relevantes, dado que os retornos para prazos tão curtos tendem a ser próximos a zero.

O resultado encontrado confirma a teoria. Em períodos de alta volatilidade, os agentes tendem a aumentar a taxa de desconto na projeção do valor das ações, reduzindo o valor das ações e, consequentemente, gerando um retorno médio negativo para o lbovespa. O movimento da Ptax é o inverso. Em períodos de maior incerteza, os agentes, principalmente internacionais, tendem a buscar proteção no dólar americano, vendendo ativos financeiros em um movimento de stop loss e adquirindo a moeda estrangeira. Esse movimento tende a refletir a alta na cotação do dólar, geralmente observado em períodos de crise.

O resultado mais interessante é a percepção de que o índice FXvol, por refletir a expectativa do mercado para a volatilidade da taxa cambial um mês a frente, apresenta evidências de ser útil como indicador antecedente para movimentos futuros da Ptax e do lbovespa. 


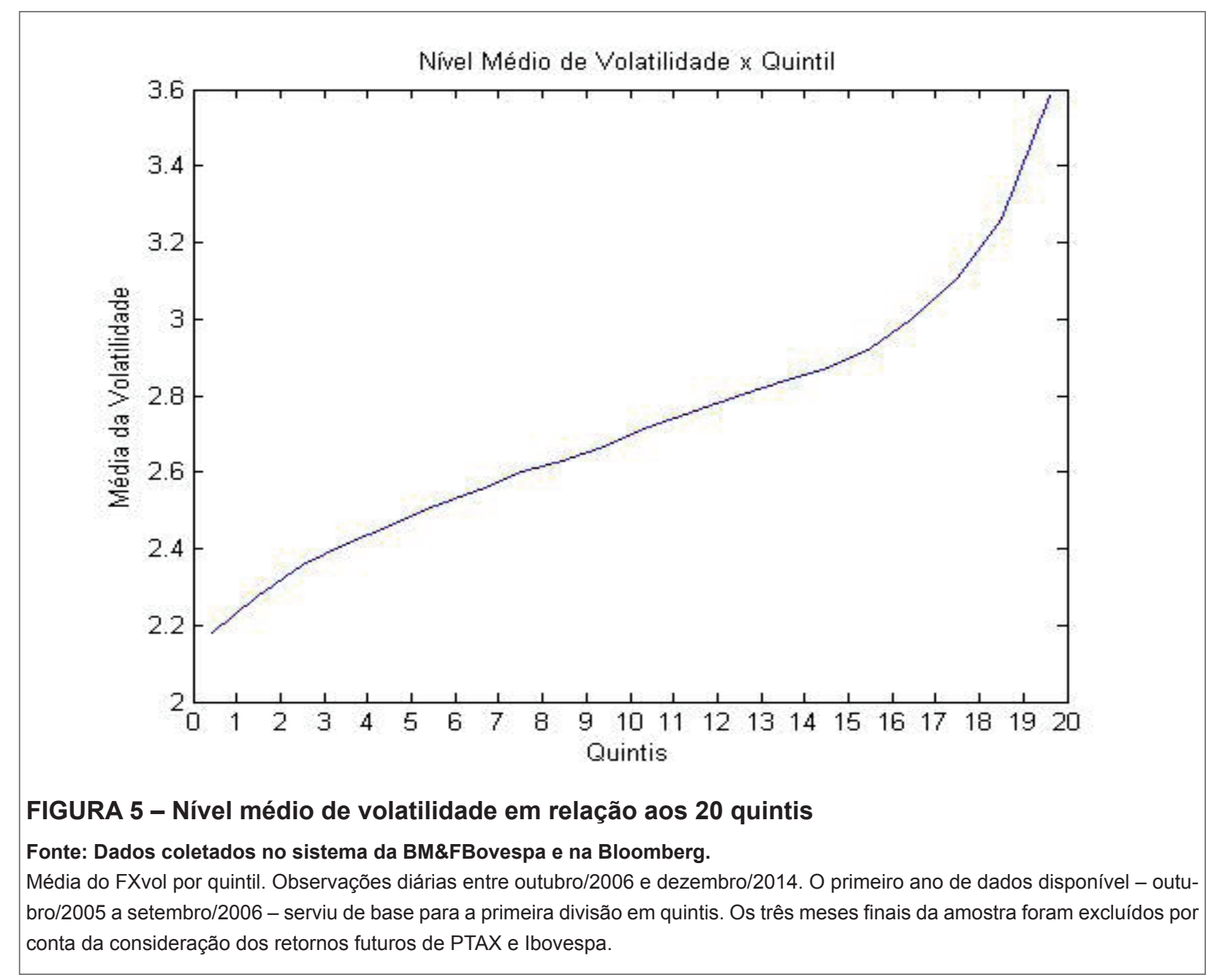

TABELA 8 - Retornos Médios observados após períodos de alta e baixa volatilidade

\begin{tabular}{l|c|c|c|c|c|c|c|c}
\hline & \multicolumn{9}{|c}{ Ptax } & \multicolumn{3}{c}{ Retornos Médios } \\
\hline & \multicolumn{9}{|c|}{ Ibovespa } \\
\hline & 1 d.u. & 10 d.u. & 21 d.u. & 63 d.u. & 1 d.u. & 10 d.u. & 21 d.u. & 63 d.u. \\
\hline & & & & & & & & \\
\hline Baixa Volatilidade & 0,00 & $-0,26$ & $-0,75$ & $-4,12$ & $-0,02$ & 0,11 & 0,49 & 3,34 \\
\hline & & & & & & & & \\
\hline Alta Volatilidade & 0,08 & 0,80 & 2,30 & 10,30 & 0,02 & $-0,02$ & $-0,61$ & $-3,34$ \\
\hline
\end{tabular}

Fonte: Dados coletados no sistema da BM\&FBovespa e na Bloomberg.

Nota: Retornos médios apresentados pela Ptax e pelo Ibovespa 1, 10, 21 e 63 d.u. após uma observação de baixa ou alta volatilidade. São classificadas como baixa volatilidade as observações classificadas entre os 5 primeiros quintis da distribuição das volatilidades diárias observadas entre outubro/2005 e novembro/2014. São classificadas como alta volatilidade as observações classificadas entre os 5 últimos quintis, no mesmo período. Valores expressos em \% ao período.

\section{Conclusão}

A pesquisa teve por objetivo investigar a relação contemporânea entre o FXvol, a Ptax e o lbovespa, bem como a capacidade do FXvol de captar a possível relação entre o nível de incerteza presente no mercado e as variações relativas futuras da taxa de câmbio e do índice de ações. Para tanto, examinou-se o índice $\mathrm{FXvol}$, registrado diariamente, para o período de outubro 
de 2005 a fevereiro de 20I5, elaborando análises descritivas, análise da assimetria na variação diária da série e as relações entre o FXvol e a Ptax e o lbovespa.

A análise de assimetria indica que o FXvol age como um captador do medo do mercado, pois retornos negativos do lbovespa estão atrelados a um aumento no índice de volatilidade cambial mais forte que a retornos positivos e a queda do índice. Esse mesmo comportamento foi visto entre o FXvol e a Ptax.

As relações entre o FXvol e a Ptax e o Ibovespa demonstram que, em média, retornos futuros de duas semanas, um mês e três meses negativos para a Ptax e positivos para o lbovespa são precedidos de períodos de baixa volatilidade, mensurada pelo $\mathrm{FXvol}$. Já períodos de retornos futuros positivos para a Ptax e negativos para o lbovespa, para os mesmos prazos, são antecedidos por períodos de alta no índice de volatilidade cambial.
Esses resultados corroboram a teoria que afirma que, em períodos de crise, as agentes ajustam as taxas de desconto dos resultados futuros dos ativos, reduzindo o preço das ações e gerando um resultado médio negativo para o lbovespa e buscam "proteção" no dólar americano, gerando uma alta no preço da moeda. Esses indícios demonstram uma boa capacidade do FXvol como indicador antecedente do comportamento, não só da taxa de câmbio, ativo com o qual tem relação direta, mas também do índice do mercado acionário, com o qual não possui nenhuma relação direta, mas uma correlação significante.

Como sugestão para a continuidade da pesquisa, seria relevante analisar se o $\mathrm{FXvol}$ aumenta a acurácia de um modelo de séries temporais da Ptax e do lbovespa.Além disso, verificar se os resultados encontrados para o índice lbovespa persistem nos índices setoriais, como, por exemplo, no Índice Industrial (INDX). 


\section{REFERÊNCIAS}

BARROS, D. Volatilidade Cambial: um obstáculo à coordenação de políticas macroeconômicas no Mercosul. A Economia em Revista, [S. I.], v. I7, n. I, p. 3 I-40, 2009.

BITTENCOURT, M.V. L.; LARSON, D. W.; THOMPSON, S. R. Impactos da volatilidade da taxa de câmbio no comércio setorial do Mercosul. Estudos Econômicos, [S. I.], v. 37, n. 4, p. 79l-816, 2007.

BROOKS, C. Introductory Econometrics for Finance. $3^{\text {rd }}$. ed. Cambridge University Press: New York, 2014.

CHEN, L.; DA, Z.; ZHAO, X.What Drives Stock Price Movements? The Review of Financial Studies, [S. I.], v. 26, n. 4, p. 84I-876, 2013.

CORRADO, C. J.; MILLER JR, T.W. Estimating expected excess returns using historical and option-implied volatility. The Journal of Financial Research, [S. I.], v. 29, n. I, p. 95- I | 2, 2006.

COUTINHO, J. R. R.; SHENG, H. H.; LORA, M. I. The use of Fx Derivatives and the Cost of Capital: Evidence of Brazilian companies. Emerging Markets Review, v. |3, n. 4, p. 4| |-423, 2012.
DARIO, A. D. G. Índice de Volatilidade para o Mercado Brasileiro de Câmbio: Fxvol. Resenha BM\&F, [S. I.], n. I72, p. 68-76, 2007.

DEMETERFI, K.; DERMAN, E.; KAMAL, M.; ZOU, J. A Guide to Volatility and Variance Swaps. Journal of Derivatives, [S. I.], v. 6, p. 9-32, 1999.

GABE, J.; PORTUGAL, M. S. Volatilidade implícita versus volatilidade estática: um exercício utilizando opções e ações da Telemar S. A. Revista Brasileira de Finanças, [S. I.], v. 2, n. I, 2004.

GIOT, P. Relationships between Implied Volatility Indexes and Stock Index Returns: are implied volatility indexes leading indicators? The Journal of Portfolio Management, [S. I.], v. 31 , n. 3, p. 92 100, 2005.

GRÔPPO, G. S. Relação dinâmica entre lbovespa e variáveis de política monetária. Revista de Administração de Empresa, [S. I.], v. 46, p. 72-85, 2006. Edição especial.

LOPES, J. L. G.; SCHIOZER, R. F.; SHENG, H. H. Hedge e Especulação com Derivativos Cambiais: evidências de operações cotidia- nas. Revista de Administração Contemporânea, [S. I.], v. I7, n. 4, p. 438-458, 2013.

PIMENTA JÚNIOR, T.; e SCHERMA, F.

R. Um Estudo da Influência entre o Dólar e o lbovespa no Período 1999-2003. Revista Eletrônica de Gestão Organizacional, [S. I.], v. 3, n. I, p. I8-25, 2005.

SILVA, F. M.; CORONEL, D. A. Análise da causalidade e cointegração entre variáveis macroeconômicas e o lbovespa. Revista de Administração FACES Journal, Belo Horizonte, v. II, n. 3, p. 3I-52, 20 I 2. WHALEY, R. E. The Investor Fear Gauge. Journal of Portfolio Management, [S. I.], n. 26, v. 3, p. I2-17, 2000.

WOO, R. K.; VICENTE, J. V. M.; BARBEDO, C. H. É possível replicar a volatilidade da taxa de câmbio com instrumentos transacionais no mercado? Revista Brasileira de Finanças, [S. I.], v. 7, n. 4, p. 485-50I, 2009.

XIN, Cheng. Three Essays on Volatility Forecasting. 2010. 109 f. Tese (Doutorado em Finanças) - Hong Kong Baptist University, Hong Kong, 2010. 